\title{
Higgsino dark matter in the MSSM
}

\author{
Antonio Delgado ${ }^{1}$ and Mariano Quirós ${ }^{2}$ \\ ${ }^{1}$ Department of Physics, University of Notre Dame, 225 Nieuwland Hall Notre Dame, Indiana 46556, USA \\ ${ }^{2}$ Institut de Física d'Altes Energies (IFAE) and BIST, Campus UAB 08193 Bellaterra, Barcelona, Spain
}

(Received 10 August 2020; accepted 6 January 2021; published 22 January 2021)

\begin{abstract}
A cosmologically stable neutral component from a nearly pure $S U(2)$ doublet, with a mass $\sim 1.1 \mathrm{TeV}$, is one appealing candidate for dark matter (DM) consistent with all direct dark matter searches. We explore this possibility in the context of the minimal supersymmetric extension of the Standard Model, with the Higgsino playing the role of DM, in theories where supersymmetry breaking is transmitted by gravitational interactions at the unification scale $M \simeq 2 \times 10^{16} \mathrm{GeV}$. We focus on the search for "light" supersymmetric spectra, which could be within reach of present and/or future colliders, in models with universal and nonuniversal Higgs and gaugino Majorana masses. The lightest supersymmetric particles of the spectrum are, by construction, two neutralinos and one chargino, almost degenerate, with a mass $\sim 1.1 \mathrm{TeV}$, and a mass splitting of a few GeV. Depending on the particular scenario the gluino can be at its experimental lower mass bound $\sim 2.2 \mathrm{TeV}$; in the squark sector, the lightest top squark can be as light as $\sim 1.6 \mathrm{TeV}$, and the lightest slepton, the right-handed stau, can have a mass as light as $1.2 \mathrm{TeV}$. The lightest neutralino can be found in the next generation of direct dark matter experimental searches. In the most favorable situation, the gluino, with some specific decay channels, could be found during the next run of the LHC, and the lightest top squark during the high-luminosity LHC run.
\end{abstract}

DOI: 10.1103/PhysRevD.103.015024

\section{INTRODUCTION}

Supersymmetry, and in particular the minimal supersymmetric extension of the Standard Model (MSSM), remains the most appealing solution to the Standard Model's naturalness problem [1,2]. In spite of all of the negative results from experimental searches, the fact that the Higgs boson was found with a mass $m_{h} \simeq 125 \mathrm{GeV}$ points toward a heavy supersymmetric spectrum, so that an irreducible little hierarchy problem is unavoidable. Supersymmetric spectra in the few- $\mathrm{TeV}$ range are still allowed by present searches at the LHC [3].

An important spin-off of the MSSM in the presence of $R$-parity conservation (which prevents baryon and lepton number violation at the perturbative level and thus proton decay) is that the lightest supersymmetric particle (LSP), if electrically neutral, is a candidate for (cold) dark matter (DM) and can give rise to the observed dark matter density after thermal freeze-out [4]. The possibility that DM is the lightest neutralino has been explored for a long time and is one of the most appealing features of the MSSM [5-18]. Given the strong bounds on the mass of supersymmetric

Published by the American Physical Society under the terms of the Creative Commons Attribution 4.0 International license. Further distribution of this work must maintain attribution to the author(s) and the published article's title, journal citation, and DOI. Funded by SCOAP ${ }^{3}$. particles and the plethora of null results from direct search experiments [3], there is a clearly preferred scenario: a nearly pure Higgsino with a mass $\sim 1.1 \mathrm{TeV}[10,19]$.

In view of the previous comments, in this paper we consider the possibility that the MSSM encompasses a supersymmetric spectrum where the LSP is a nearly pure Higgsino with a mass $\sim 1.1 \mathrm{TeV}$. As the MSSM spectrum largely depends on the supersymmetry-breaking mechanism and the solution to the supersymmetric $\mu$ problem (generation of the $\mu$ term in the Higgs superpotential), we work with models of gravity mediation of supersymmetry breaking, where the $\mu$ term can be generated through a nonrenormalizable contribution to the Kähler potential, i.e., the Giudice-Masiero mechanism [20]. For these models the scale $M$ at which supersymmetry is broken (i.e., the scale at which the soft-breaking masses are generated) is identified with the scale where gauge couplings unify, i.e., the unification scale $M \simeq 2 \times 10^{16} \mathrm{GeV}$ [2]. These models have minimal supergravity as the ultraviolet (UV) completion and are inspired by (and obtained from) string constructions [21]. In these scenarios the soft-breaking masses do depend on the localization of Standard Model fields in the extra dimensions, and thus there are two simple scenarios for scalars: i) models where, at the unification scale, the Higgs and sfermion masses are equal (dubbed universal Higgs mass models), and ii) models where the Higgs and sfermion masses are different (dubbed nonuniversal Higgs mass models). On the other hand, as gaugino 
Majorana masses $\left(M_{a}\right)$ evolve as the corresponding gauge couplings $\left(\alpha_{a}\right)$, it is usually assumed that gaugino Majorana masses unify at the gauge coupling unification scale, although in supergravity models these masses depend on nonrenormalizable $F$-density couplings in the gauge sector and can be different.

To summarize, the main purpose of this paper is to make predictions on the supersymmetric mass spectra of models where a $1.1 \mathrm{TeV}$ Higgsino is the LSP and supersymmetry is broken at the unification scale, which can be useful to guide experimental searches and, in particular, to look for the existence of supersymmetric spectra that will be within reach of present or future colliders. Given the present bounds on supersymmetric masses, we do not pay particular attention to the issue of fine-tuning, ${ }^{1}$ but rather to the possibility of experimental detection of the supersymmetric spectra. For this reason we abandon the criterion of Majorana mass unification and consider cases where the gluino is on the verge of experimental detection. We will see that with a lighter gluino the renormalization over the other supersymmetric parameters is smaller and the resulting squark spectra are lighter than those with a heavy gluino.

The contents of this paper are as follows. In Sec. II the conditions for electroweak and supersymmetry breaking are summarized for the scalar sector. In Sec. III the conditions for the LSP to be an almost pure Higgsino with a mass of $\sim 1.1 \mathrm{TeV}$ are established. The spectrum of charginos and neutralinos is fully determined, with all generality, after the conditions from the XENON1T direct searches are imposed. In Sec. IV we obtain predictions for supersymmetric spectra for different scenarios of supersymmetry breaking. In particular, scenarios of universal and nonuniversal Higgs masses, at the unification scale $M$, as well as those of universal and nonuniversal gaugino masses are studied separately. In Sec. V we comment on the experimental signatures at hadron colliders of the considered scenarios. Finally, we present our conclusions and outlook in Sec. VI.

\section{ELECTROWEAK AND SUPERSYMMETRY BREAKING}

In the MSSM electroweak symmetry breaking (EWSB) is achieved by means of two-Higgs doublets $H_{U}$ and $H_{D}$ whose vacuum expectation values give a mass to up-like quarks, and down-like quarks and charged leptons, respectively. The corresponding superfields $\mathcal{H}_{U}$ and $\mathcal{H}_{D}$ appear in the superpotential as $W=\mu \mathcal{H}_{U} \cdot \mathcal{H}_{D}$, which gives a supersymmetric mass to Higgs bosons and Higgsinos. Moreover, through the process of supersymmetry breaking, the Higgs sector acquires soft-breaking masses as

\footnotetext{
${ }^{1}$ An analysis based on fine-tuning criteria was done in Ref. [22].
}

$$
-\mathcal{L}_{\text {soft }}=m_{H_{U}}^{2}\left|H_{U}\right|^{2}+m_{H_{D}}^{2}\left|H_{D}\right|^{2}+\left(b H_{U} \cdot H_{D}+\text { H.c. }\right) .
$$

After imposing EW breaking at a low scale $\mathcal{Q}_{0}$ as $\left\langle H_{U}\right\rangle=v_{U},\left\langle H_{D}\right\rangle=v_{D}$, with $t_{\beta} \equiv \tan \beta=v_{U} / v_{D}$, the equations of motion (EoM) are

$$
\begin{aligned}
m_{H_{U}}^{2} & =-\left(\mu^{2}+\frac{1}{2} m_{Z}^{2}\right) \frac{t_{\beta}^{2}-1}{t_{\beta}^{2}}+m_{H_{D}}^{2} / t_{\beta}^{2}, \\
\sin 2 \beta & =\frac{2 b}{m_{H_{U}}^{2}+m_{H_{D}}^{2}+2 \mu^{2}},
\end{aligned}
$$

where all parameters are considered at the scale $\mathcal{Q}_{0}$.

As is obvious from the EoM (2.2), EWSB in the MSSM requires supersymmetry breaking. We will assume that at the high scale $M$ (the supersymmetry breaking scale) softbreaking parameters are generated for the Higgses, gauginos, and (third-generation) squarks as

$m_{H_{U}}^{0}, \quad m_{H_{D}}^{0}, \quad m_{Q}^{0}, \quad m_{U}^{0}, \quad m_{D}^{0}, \quad A_{t}^{0}, \quad M_{a}^{0} \quad(a=1,2,3)$,

where the zero upper index indicates that the corresponding parameter is evaluated at the scale $M$. Using the renormalization group evolution of the parameters from the high scale $M$ to the low scale $\mathcal{Q}_{0}$, the values specified in Eq. (2.3) should be considered as boundary conditions. We will specify the corresponding parameter values at the low scale $\mathcal{Q}_{0}$ with no upper index, i.e., $m_{X}$.

Hereafter, we consider gravity-mediated supersymmetry breaking, for which the supersymmetry-breaking scale is at the unification scale $M \sim 2 \times 10^{16} \mathrm{GeV}$, and the softbreaking terms depend on the superpotential and Kähler potential dependences of the superfield $\mathcal{X}$ which spontaneously break supersymmetry through its $F_{X}$ term. This allows for many different possibilities or relationships between the supersymmetry-breaking parameters in Eq. (2.3) [2]. Moreover, in supergravity models the $\mu$ and $b$ terms can be obtained via the Giudice-Masiero mechanism [20] through nonrenormalizable contributions to the Kähler potential as

$K=\frac{\lambda_{\mu}}{M_{P}} \mathcal{H}_{U} \cdot \mathcal{H}_{D} \mathcal{X}^{\dagger}+\frac{\lambda_{b}}{M_{P}^{2}} \mathcal{H}_{U} \cdot \mathcal{H}_{D}|\mathcal{X}|^{2}+$ H.c.

leading to

$$
\mu^{0}=\frac{\lambda_{\mu}}{M_{P}} F_{X}^{\dagger}, \quad b^{0}=\frac{\lambda_{b}}{M_{P}^{2}}\left|F_{X}\right|^{2} .
$$

As the values of the $\mu^{0}$ and $b^{0}$ terms at the scale $M$ depend on unknown parameters of the UV supergravity completion, we 
can consider their values at the low scale $\mathcal{Q}_{0}$ in the EoM (2.2) as free parameters: $\mu$ and $b$.

Moreover, the gaugino Majorana mass entry $M_{a b}$ is given in terms of the Kähler potential $K$, superpotential $W$, and gauge kinetic function $f_{a b}\left(\phi^{i}\right)$ (an analytic function of the scalar fields $\phi^{i}$ which transforms under the gauge group as the symmetric product of adjoint representations) as

$$
\mathcal{M}_{a b}=\frac{1}{2 \operatorname{Re} f_{a b}} e^{-G / 2} G^{i}\left(G^{-1}\right)_{i j} \frac{\partial f_{a b}^{*}}{\partial \phi_{j}},
$$

where $G=K+W$. Depending on the particular UV (supergravity) completion of the model, the gaugino mass spectrum can behave in different ways at the unification scale. A survey of nonuniversal gaugino mass models from grand unified and string models can be found in Ref. [23].

Here we mainly study the case of intermediate $\tan \beta$, $1 \ll \tan \beta \ll m_{t} / m_{b}$, and thus we neglect all Yukawa couplings except the top-quark one. Then, we can write the soft-breaking terms that appear in the first equation of Eq. (2.2) in terms of their values at $M$. In Refs. [24,25] we integrated the renormalization group equations (RGEs) between the high scale $M$ and low scale $\mathcal{Q}_{0}$. For cases where the hypercharge $D$ term vanishes, i.e., $m_{H_{U}}^{2}-m_{H_{D}}^{2}+$ $\sum_{a}\left(m_{Q}^{2}-2 m_{U}^{2}+m_{D}^{2}-m_{L}^{2}+m_{E}^{2}\right)_{a}=0$ (where $a$ is a generation index), an equality that is RGE invariant (and which will cover all cases considered in this paper), the soft-breaking terms sensitive to the top Yukawa coupling at the scale $\mathcal{Q}_{0}$ are linear combinations of the parameters at the scale $M:\left(m_{Q}^{0}\right)^{2},\left(m_{U}^{0}\right)^{2},\left(m_{H_{U}}^{0}\right)^{2}, M_{a}^{0} M_{b}^{0}, M_{a}^{0} A_{t}^{0}$, and $\left(A_{t}^{0}\right)^{2}$. In particular, we can write

$$
\begin{aligned}
m_{H_{U}}^{2}= & \left(m_{H_{U}}^{0}\right)^{2}+\eta_{Q}\left[\left(m_{Q}^{0}\right)^{2}+\left(m_{U}^{0}\right)^{2}+\left(m_{H_{U}}^{0}\right)^{2}\right] \\
& +\eta_{a} \sum_{a}\left(M_{a}^{0}\right)^{2}+\eta_{a b} \sum_{a \neq b} M_{a}^{0} M_{b}^{0} \\
& +\sum_{a} \eta_{a A} M_{a}^{0} A_{t}^{0}+\eta_{A}\left(A_{t}^{0}\right)^{2}
\end{aligned}
$$

where all of the coefficients $\eta_{X}=\eta_{X}\left(\mathcal{Q}_{0}, M\right)$ are functions of the high scale $M$ and the low scale $\mathcal{Q}_{0}$ (fitted in Ref. [24]) and are used throughout this work. As for the other breaking parameter in Eq. (2.2), $m_{H_{D}}^{2}$, as we neglect the bottom Yukawa coupling, it is renormalized by gauge interactions so that in the one-loop approximation it can be given by [2]

$$
\begin{aligned}
m_{H_{D}}^{2}= & \left(m_{H_{D}}^{0}\right)^{2}+\frac{3}{2}\left(1-\frac{\alpha_{2}^{2}\left(\mathcal{Q}_{0}\right)}{\alpha_{2}^{2}(M)}\right)\left(M_{2}^{0}\right)^{2} \\
& +\frac{1}{22}\left(1-\frac{\alpha_{1}^{2}\left(\mathcal{Q}_{0}\right)}{\alpha_{1}^{2}(M)}\right)\left(M_{1}^{0}\right)^{2} .
\end{aligned}
$$

Concerning the second equation in Eq. (2.2), the value of $t_{\beta}$ is determined by the soft-breaking parameter $b$. In the limit of large $t_{\beta}$, and using the first equation in Eq. (2.2), it is given by

$$
t_{\beta} \simeq \frac{m_{H_{D}}^{2}+\mu^{2}-m_{Z}^{2} / 2}{b} \simeq \frac{m_{H_{D}}^{2}}{b},
$$

where in the last equality we assume that the soft-breaking masses are much larger than $\mu$. So, as is natural in the mechanism of Eq. (2.5), for $\lambda_{b}=\mathcal{O}\left(\lambda_{\mu}\right)$ we should get $b \simeq \mu^{2}$ and for $m_{H_{D}} \gg|\mu|$ we should get $t_{\beta} \gg 1$. In view of our ignorance of the UV completion of the model, we consider $t_{\beta}$ as a free parameter.

\section{THE DARK MATTER SECTOR}

The Higgs boson's supersymmetric partners, $\tilde{H}_{U}$ and $\tilde{H}_{D}$, along with the supersymmetric partners of the $S U(3) \otimes S U(2)_{L} \otimes U(1)_{Y}$ gauge bosons, $\tilde{g}, \tilde{W}$, and $\tilde{B}$, make a set of four neutral Majorana fermions (neutralinos) $\chi_{1,2,3,4}^{0}$, two charged fermions (charginos) $\chi_{1,2}^{ \pm}$, and eight gluinos $\tilde{g}$.

Charginos and neutralinos get masses from the superpotential $W=\mu \mathcal{H}_{U} \cdot \mathcal{H}_{D}$, from the soft-breaking Majorana masses $M_{1,2}$ for $\tilde{B}$ and $\tilde{W}$, respectively, and from the electroweak breaking. The mass matrices for neutralinos and charginos are then given by

$$
\begin{gathered}
\mathcal{M}_{0}=\left[\begin{array}{cccc}
M_{1} & 0 & -c_{\beta} s_{W} m_{Z} & s_{\beta} s_{W} m_{Z} \\
0 & M_{2} & c_{\beta} c_{W} m_{Z} & -s_{\beta} c_{W} m_{Z} \\
-c_{\beta} s_{W} m_{Z} & c_{\beta} c_{W} m_{Z} & 0 & -\mu \\
s_{\beta} s_{W} m_{Z} & -s_{\beta} c_{W} m_{Z} & -\mu & 0
\end{array}\right], \\
\mathcal{M}^{ \pm}=\left[\begin{array}{cc}
0 & X^{T} \\
X & 0
\end{array}\right], \\
X=\left[\begin{array}{cc}
M_{2} & \sqrt{2} s_{\beta} m_{W} \\
\sqrt{2} c_{\beta} m_{W} & \mu
\end{array}\right],
\end{gathered}
$$

where again all parameters are evaluated at the scale $\mathcal{Q}_{0}$, $s_{W}=\sin \theta_{W}$, and so on.

One of the most appealing features of the MSSM (in the presence of $R$ parity, a symmetry preventing proton decay) is its ability to provide a viable DM candidate as the LSP, in particular the lightest neutralino $\chi_{1}^{0}$. However, from the plethora of DM searches in direct-detection experiments, large regions of the parameter space of neutralino DM have been excluded [26]. One scenario that is still viable is a nearly pure Higgsino with a mass $\sim 1.1 \mathrm{TeV}[10,19]$. This happens whenever $|\mu| \ll M_{1}, M_{2}$ and $|\mu| \simeq 1.1 \mathrm{TeV}$. In this case the coupling of $\chi_{1}^{0}$ with the proton comes at tree level from the coupling with the Higgs $h, \bar{\chi}_{1}^{0} \chi_{1}^{0} h$, induced by the mixing of Higgsinos with gauginos, leading to spin-independent cross sections with heavy nuclei. 

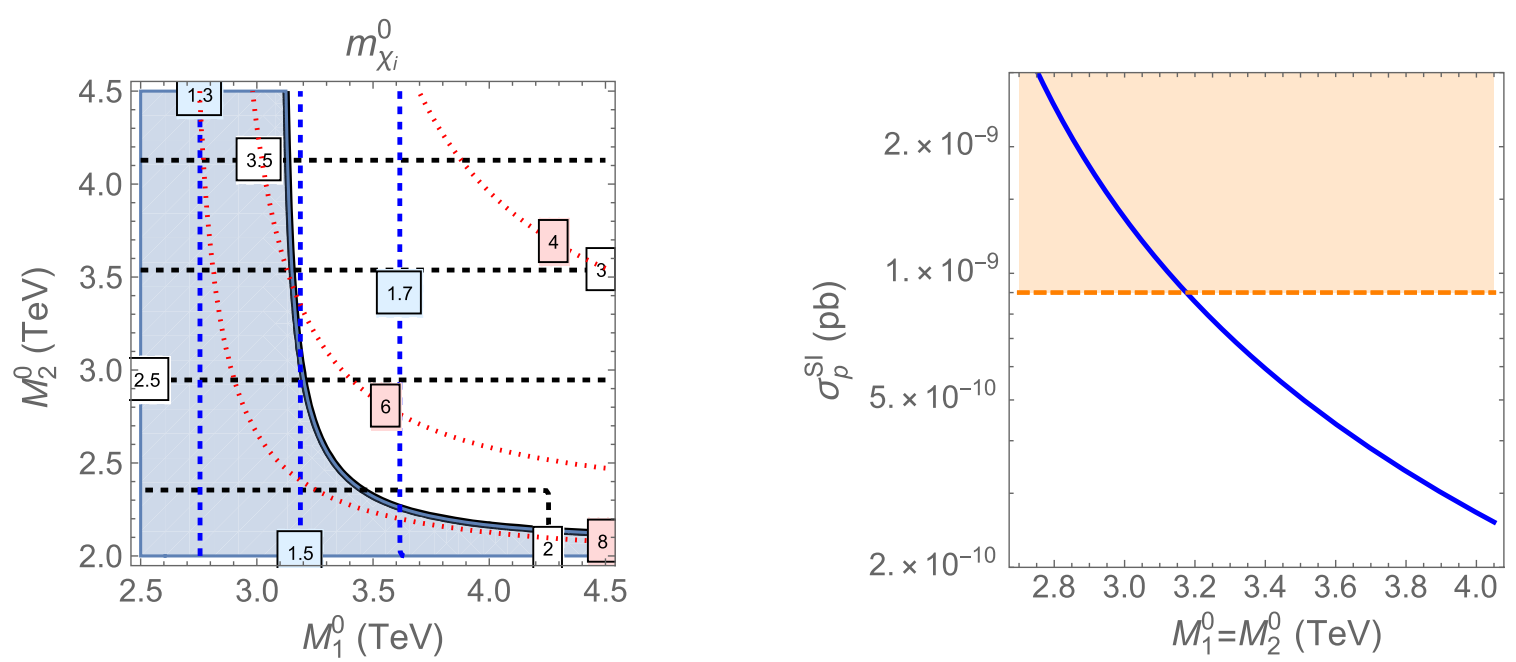

FIG. 1. Left: the shaded region is forbidden by the spin-independent cross section for $\bar{\chi}_{1}^{0} \chi_{1}^{0}$-proton in the plane $\left(M_{1}^{0}, M_{2}^{0}\right)$. Vertical (horizontal) dashed lines are mass values of $\chi_{3}^{0}\left(\chi_{4}^{0}\right)$ in TeV. Dotted lines are $m_{\chi_{2}^{0}}-m_{\chi_{1}^{0}}$ in GeV. Right: plot of $\sigma_{p}^{\mathrm{SI}}$ (in pb) as a function of $M_{1}^{0}=M_{2}^{0}$ in $\mathrm{TeV}$.

The spin-independent cross section with the proton is bounded by the XENON1T experiment [26] which, for $m_{\chi_{1}^{0}} \simeq 1 \mathrm{TeV}$, obtained the $90 \%$ C.L. bound $\sigma_{p}^{\mathrm{SI}} \lesssim$ $9 \times 10^{-10} \mathrm{pb}$, which we will hereafter consider as a conservative limit.

In the left panel of Fig. 1 we show the (thick solid) contour line of $\sigma_{p}^{S I}=9 \times 10^{-10} \mathrm{pb}$ in the plane of Majorana masses $\left(M_{1}^{0}, M_{2}^{0}\right)$. Thus, the shaded region is forbidden by the XENON1T experiment [26]. The mass spectrum is then as follows. i) The LSP $\chi_{1}^{0}$ has a mass $\sim 1.1 \mathrm{TeV}$ in the entire region shown, while the next to lightest neutralino $\chi_{2}^{0}$ has a mass $m_{\chi_{2}^{0}}$ that is larger than $m_{\chi_{1}^{0}}$ by a few $\mathrm{GeV}$, as we can see from the dotted contour lines. ii) The heavy states $\chi_{3}^{0}\left(\chi_{4}^{0}\right)$, with mass labels in $\mathrm{TeV}$, are shown by the vertical (horizontal) dashed lines. Therefore, we can see that $\chi_{1}^{0}$ and $\chi_{2}^{0}$ are almost degenerate in mass around $1.1 \mathrm{TeV}$, while $m_{\chi_{3}}$ depends mainly on $M_{1}^{0}$, and $m_{\chi_{4}^{0}}$ depends mainly on $M_{2}^{0}$. In the allowed region we infer that $M_{1}^{0} \gtrsim 3 \mathrm{TeV}$ which corresponds to $m_{\chi_{3}^{0}} \gtrsim 1.5 \mathrm{TeV}$, while if we want to stick to the lowest possible values of $m_{\chi_{3}^{0}}$ we need to consider the region where $M_{2}^{0} \gtrsim M_{1}^{0}$, although the larger $M_{2}^{0}$, the larger $m_{\chi_{4}^{0} \text {. We conclude from this analysis }}$ that, in order to obtain the lightest possible neutralino spectrum, we should consider that $M_{1}^{0} \sim M_{2}^{0}$. As $M_{a}$ ( $a=1,2,3)$ evolves with the RGE as the couplings $\alpha_{a}$, it is a sensible condition to consider the unification condition $M_{1}^{0}=M_{2}^{0}$, as we do hereafter.

In the right panel of Fig. 1 we consider the case where $M_{1}^{0}=M_{2}^{0}$ and plot the spin-independent cross section $\sigma_{p}^{\mathrm{SI}}$ as a function of $M_{1}^{0}=M_{2}^{0}$. We also plot the exclusion region from the XENON1T experimental results which translates into the lower bound $M_{1}^{0}=M_{2}^{0} \gtrsim 3.2 \mathrm{TeV}$ at $90 \%$ C.L. The mass spectra for neutralinos (charginos) are plotted in the left (right) panel of Fig. 2 as solid lines, while the mass differences $m_{\chi_{2}^{0}}-m_{\chi_{1}^{0}}$ (left panel) and $m_{\chi_{1}^{ \pm}}-m_{\chi_{1}^{0}}$ (right panel) in $\mathrm{GeV}$ are shown as dashed lines. Using the direct-detection cross section bound from the right panel of Fig. 1 we obtain the following restrictions on the neutralino and chargino running masses:

$$
\begin{array}{ll}
m_{\chi_{1}^{0}} \simeq 1.1 \mathrm{TeV}, \quad & m_{\chi_{3}^{0}} \gtrsim 1.5 \mathrm{TeV}, \\
m_{\chi_{4}^{0}} \gtrsim 2.7 \mathrm{TeV}, & m_{\chi_{2}^{0}}-m_{\chi_{1}^{0}} \lesssim 6.3 \mathrm{GeV}, \\
m_{\chi_{2}^{ \pm}} \gtrsim 2.7 \mathrm{TeV}, & m_{\chi_{1}^{ \pm}}-m_{\chi_{1}^{0}} \lesssim 3.5 \mathrm{GeV} .
\end{array}
$$

In the following, in order to minimize the neutralino and chargino mass spectra, we will consider the benchmark case defined by

$$
M_{1}^{0}=M_{2}^{0} \simeq 3|\mu| \simeq 3.3 \mathrm{TeV},
$$

which generates the spectrum given by the lower bounds in Eq. (3.3). Larger values of $M_{1}^{0}=M_{2}^{0}$ could be equally well considered but they would lead to heavier spectra.

\section{SCENARIOS OF SUPERSYMMETRY BREAKING}

At the low scale $\mathcal{Q}_{0}$, the soft-breaking masses $m_{Q}$ and $m_{U}$ and the mixing term $A_{t}$ can be obtained by integrating the RGE, and they were fitted in Ref. [25]. After imposing the EoM (2.2), one gets 

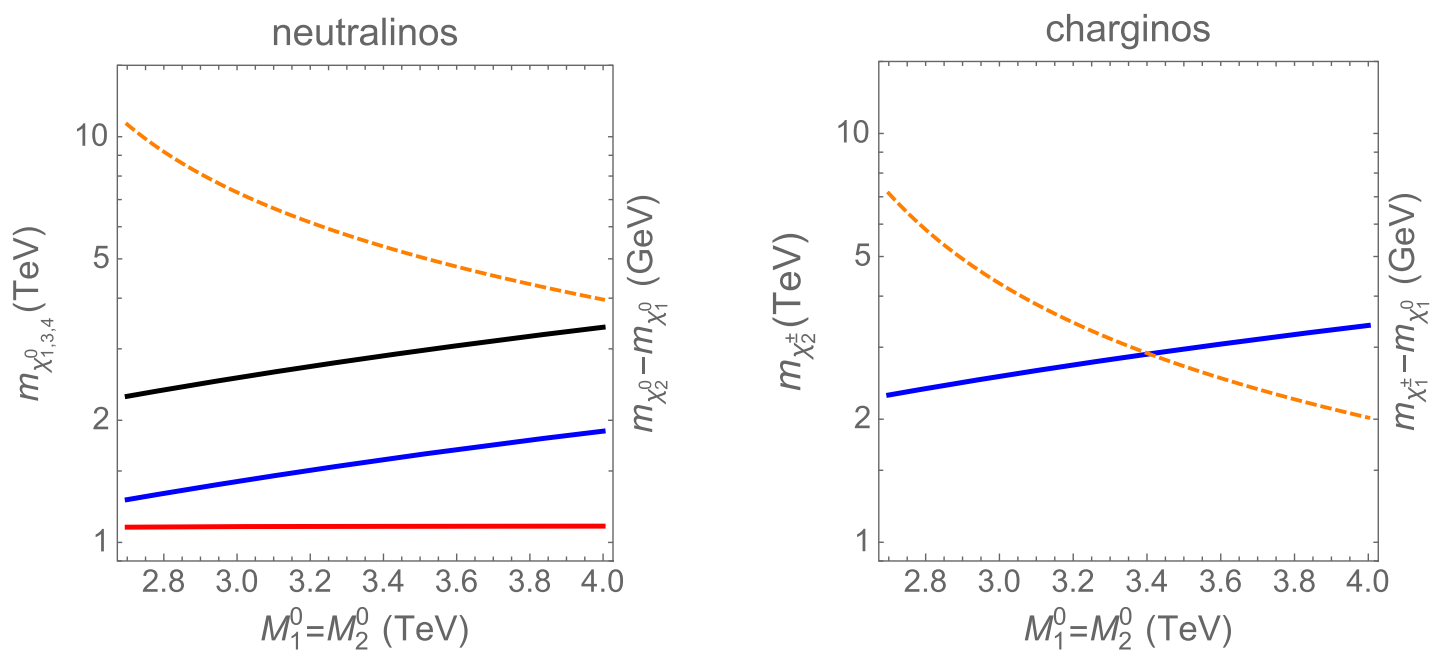

FIG. 2. Mass spectra (in TeV) for neutralinos (left) and charginos (right) (solid lines) for $M_{1}^{0}=M_{2}^{0}$ and $\mu=1.1$ TeV. The mass differences $m_{\chi_{2}^{0}}-m_{\chi_{1}^{0}}$ (left) and $m_{\chi_{1}^{ \pm}}-m_{\chi_{1}^{0}}$ (right) are in GeV.

$$
\begin{aligned}
m_{Q}^{2} & =\left(m_{Q}^{0}\right)^{2}+\sum_{a} d_{a} f_{a} \cdot\left(M_{a}^{0}\right)^{2}+\frac{1}{3} F, \\
\left(d_{1}, d_{2}, d_{3}\right) & =\left(-\frac{1}{15}, 1, \frac{8}{3}\right) \\
m_{U}^{2} & =\left(m_{U}^{0}\right)^{2}+\sum_{a} c_{a} f_{a} \cdot\left(M_{a}^{0}\right)^{2}+\frac{2}{3} F, \\
\left(c_{1}, c_{2}, c_{3}\right) & =\left(\frac{1}{3},-1, \frac{8}{3}\right)
\end{aligned}
$$

where the functions $F$ and $f_{a}$ are defined by

$$
\begin{gathered}
F=-\left(m_{H_{U}}^{0}\right)^{2}-\left(\mu^{2}+\frac{1}{2} m_{Z}^{2}\right) \frac{t_{\beta}^{2}-1}{t_{\beta}^{2}}+\frac{m_{H_{D}}^{2}}{t_{\beta}^{2}}, \\
f_{a}=\frac{1}{b_{a}} \frac{\alpha_{a}^{2}(M)-\alpha_{a}^{2}\left(\mathcal{Q}_{0}\right)}{\alpha_{a}^{2}(M)}, \\
\left(b_{1}, b_{2}, b_{3}\right)=\left(\frac{33}{5}, 1,-3\right)
\end{gathered}
$$

where $m_{H_{D}}$ is given in Eq. (2.8). In the same way, the mixing parameter at the low scale can be written as

$$
A_{t}=\sum_{a} \gamma_{a} M_{a}^{0}+\gamma_{A} A_{t}^{0},
$$

where the coefficients $\gamma_{a}$ and $\gamma_{A}$ are determined numerically and were fitted in Ref. [25].

In this paper we want to find the region of parameters in Eq. (2.3) that is consistent with a nearly pure Higgsino with a mass of $\sim 1.1 \mathrm{TeV}$ being the LSP and a good DM candidate, and that satisfies the EoM (2.2) and all present experimental constraints. We will concentrate on two

general models: i) the case of universal Higgs masses (UHM), a very popular model inspired by supergravity/ superstring constructions (also dubbed the constrained MSSM), and ii) a model where we make a separation of soft-breaking masses in the Higgs and sfermion sectors, which is motivated by string constructions if both sectors are differently located in the higher-dimensional compact space, which is dubbed nonuniversal Higgs masses [27]. In both cases we separately consider the case where all Majorana gaugino masses are unified at the high scale and the case of nonuniversal gaugino masses [28], where we concentrate on the phenomenologically interesting case where only the gluino mass does not unify with the electroweakino masses at the high scale $M$, such that the gluino is on the verge of experimental detection at the LHC.

\section{A. Universal Higgs masses}

In this section we assume the case of UHM so that the boundary conditions for the scalar sector are

$m_{Q}^{0}=m_{U}^{0}=m_{D}^{0}=m_{L}^{0}=m_{E}^{0}=m_{H_{U}}^{0}=m_{H_{D}}^{0} \equiv m_{0}$,

where only the third-generation squark masses are relevant for our study.

\section{Universal gaugino masses}

As for the Majorana gaugino masses, we first assume the case of universal gaugino masses, i.e., Majorana masses which unify at the high (unification) scale $M$ as

$$
M_{1}^{0}=M_{2}^{0}=M_{3}^{0} \equiv m_{1 / 2},
$$

in which case we have as free parameters in our model $\left(m_{0}, m_{1 / 2}, A_{t}^{0}, t_{\beta}\right)$ once we have fixed $\mu \simeq 1.1 \mathrm{TeV}$. 

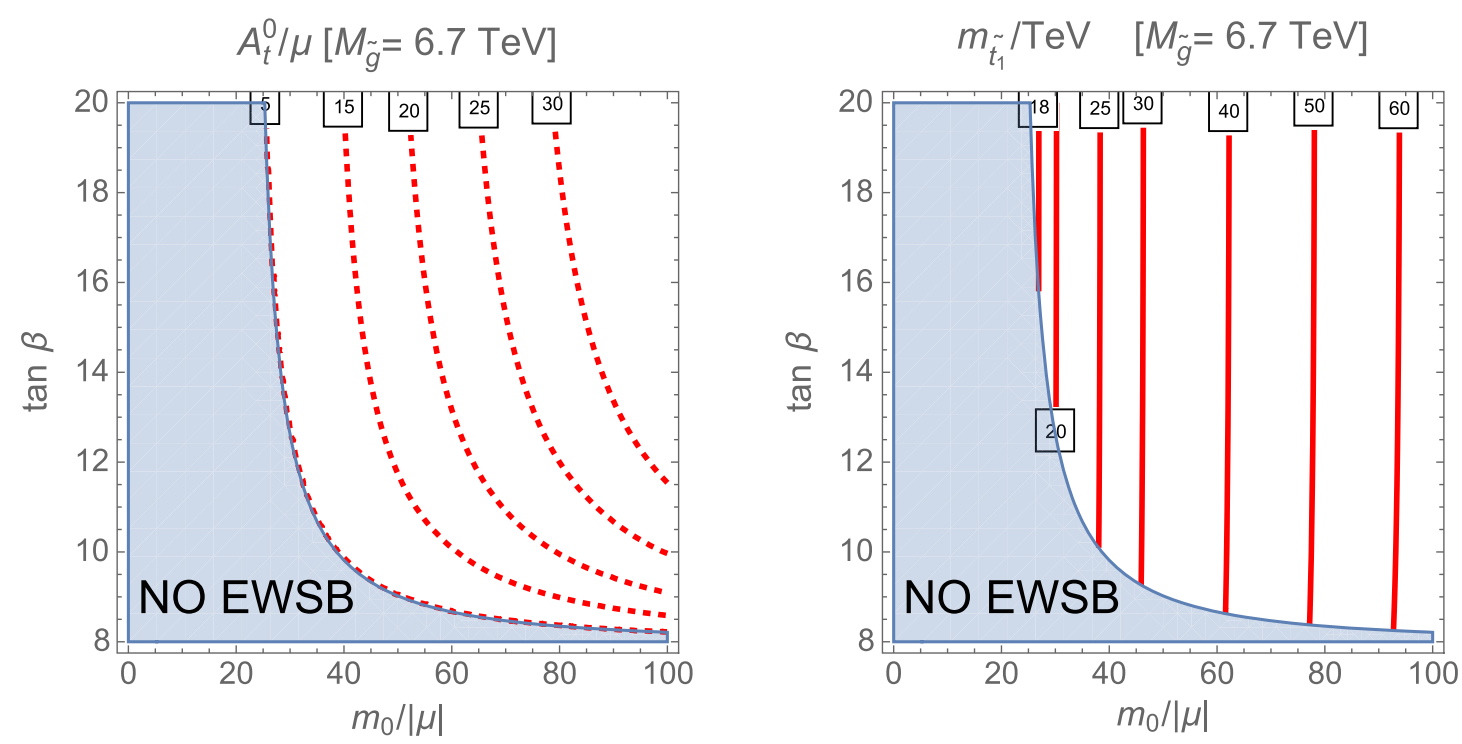

FIG. 3. Left: contour (dashed) lines of $A_{t}^{0} / \mu$ in the plane $\left(m_{0} /|\mu|, t_{\beta}\right)$ satisfying the EoM (2.2) for universal gaugino masses. The shaded region is forbidden by the EWSB condition. Right: the same for contour (solid) lines of the lightest top squark running mass $m_{\tilde{t}_{1}}$ in $\mathrm{TeV}$.

Moreover, as we want to minimize the mass of charginos and neutralinos as much as possible, we adopt the value

$$
m_{1 / 2}=3 \mu
$$

such that the mass spectrum of neutralinos and charginos is essentially given by the lower bounds in Eq. (3.3), while the gluino running mass at the low scale $\mathcal{Q}_{0}$ is $M_{\tilde{g}} \simeq 6.7 \mathrm{TeV}$, which is out of reach of LHC experimental searches. The remaining three parameters $\left(m_{0}, A_{t}^{0}, t_{\beta}\right)$ have to satisfy the EoM (2.2). The result is shown in the left panel of Fig. 3 where we show the contour lines of $A_{t}^{0} / \mu$ (red dashed lines) in the plane $\left(m_{0} /|\mu|, t_{\beta}\right)$. The shaded region corresponds to values of the parameters where the EoM is not satisfied for real values of the parameters, and thus there is no EWSB.

As we can see, the EWSB condition translates into an absolute lower bound on the parameter $t_{\beta}$ as $t_{\beta} \gtrsim 8$. Moreover, using the expressions for $m_{Q}, m_{U}$, and $A_{t}$ in Eqs. (4.1), (4.2), and (4.5), respectively, one can easily compute the (running) mass spectrum for top squarks $\left(\tilde{t}_{1}, \tilde{t}_{2}\right)$, where we use the convention that $\tilde{t}_{1}$ is the lightest top squark. Contour lines of $m_{\tilde{t}_{1}}$ are provided (red solid lines) in the right panel of Fig. 3, where we see that the solution with light top squarks is prevented by the EoM. In fact, we see that the EWSB condition implies the lower bound $m_{\tilde{t}_{1}} \gtrsim 18 \mathrm{TeV}$. As a consequence, this scenario predicts superheavy masses in the sfermion and gluino sectors, completely out of reach of future searches at the LHC. Furthermore, as the values of the mixing parameters are tiny compared mainly with the values of top squark masses, we can conclude that the mixing parameter at the low scale $\mathcal{Q}_{0}$ is negligible compared with the values of the relevant supersymmetric masses. ${ }^{2}$ Due to the heavy spectrum, this scenario might be in tension with the correct value of the Higgs mass (see Ref. [29] for more details).

\section{Nonuniversal gaugino masses}

As we have seen that, for universal gaugino masses, the gluino is heavy and out of reach of LHC experimental searches, we now explore a scenario where the gluino is on the verge of experimental detection, which is the case if the gluino Majorana mass is different at the high scale $M$ from the bino and wino Majorana masses. In particular, we assume Eq. (3.4) for $M_{1,2}^{0}$, and a value for $M_{3}^{0}$ corresponding to a running gluino mass, at the low scale, $\mathcal{Q}_{0}$ of $2.2 \mathrm{TeV}$, i.e.,

$$
M_{1}^{0}=M_{2}^{0}=3 \mu, \quad M_{\tilde{g}}=2.2 \mathrm{TeV},
$$

in which case the free parameters of the model are still $\left(m_{0}, A_{t}^{0}, t_{\beta}\right)$ as in the previous case of universal gaugino masses, but of course with a different realization of the EWSB conditions in the gaugino sector.

After imposing the EoM (2.2) the values of the parameters are provided in the left panel of Fig. 4, which shows the (dashed) contour lines of $A_{t}^{0} / \mu$ in the plane $\left(m_{0} /|\mu|, t_{\beta}\right)$. We see that for large values of $m_{0}$ there is still the lower bound $t_{\beta} \gtrsim 8$, while there is no bound on $t_{\beta}$ for small values of $m_{0}$. Similarly, the (solid) contour lines of $m_{\tilde{t}_{1}}$ in TeV are shown in the right panel of Fig. 4. Moreover, from the right panel of Fig. 4 we see that there is no lower bound on the

\footnotetext{
${ }^{2}$ In particular, we get that in the considered range $\left|A_{t}-\mu / t_{\beta}\right|<0.1 \sqrt{m_{\tilde{t}_{1}} m_{\tilde{t}_{2}}}$.
} 

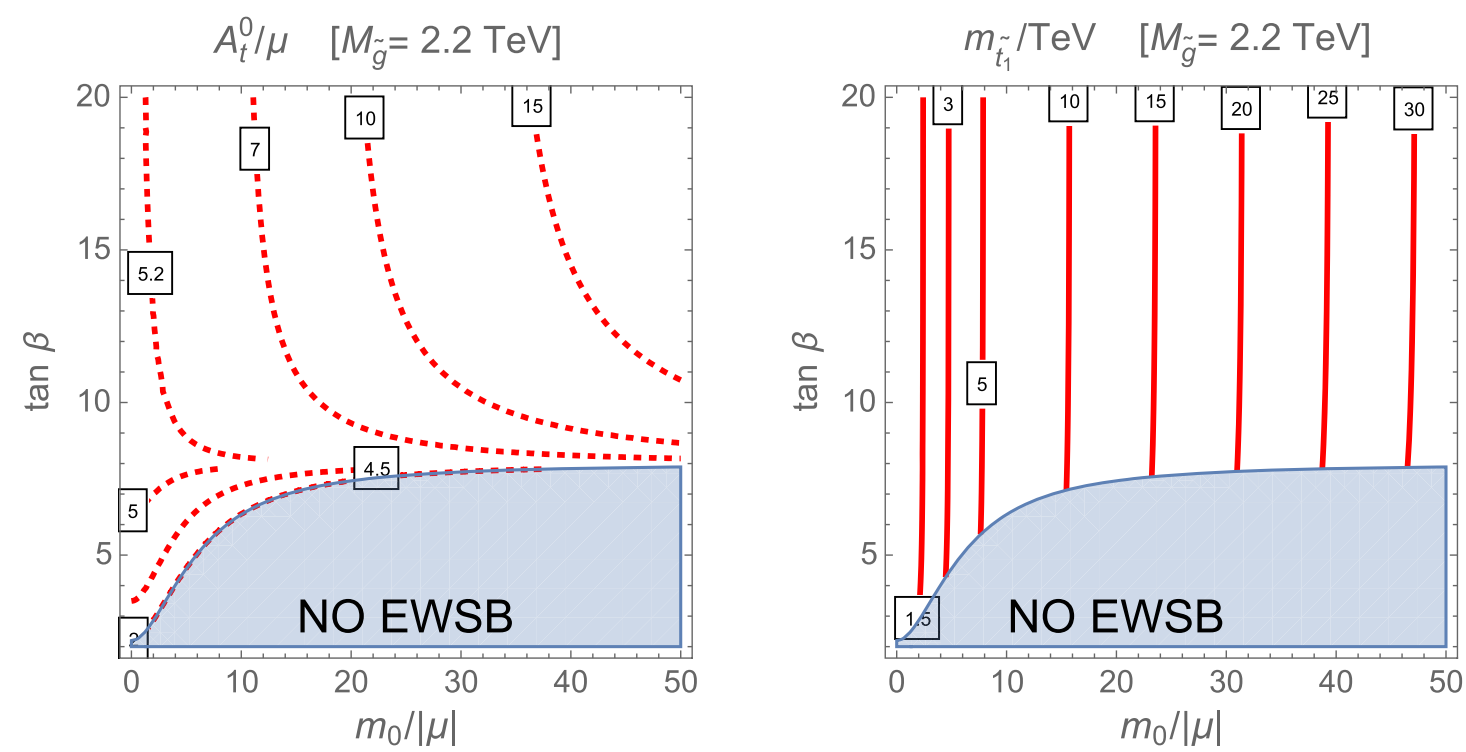

FIG. 4. Left: contour (dashed) lines of $A_{t}^{0} / \mu$ in the plane $\left(m_{0} /|\mu|, t_{\beta}\right)$ satisfying the EoM (2.2) for nonuniversal gaugino masses such that $M_{\tilde{g}}=2.2 \mathrm{TeV}$. The shaded region is forbidden by the EWSB condition. Right: the same for contour (solid) lines of the lightest top squark running mass $m_{\tilde{t}_{1}}$ in $\mathrm{TeV}$.

value of $m_{\tilde{t}_{1}}$, as there is no experimental bound on $m_{\tilde{t}_{1}}$ for the value of the LSP mass, $m_{\tilde{\chi}_{1}^{0}} \simeq 1.1 \mathrm{TeV}$ [30]. ${ }^{3}$ However, we find that in the entire region of parameter space the mixing in the top squark sector is small, $\left|A_{t}-\mu / t_{\beta}\right| \lesssim$ $0.2 \sqrt{m_{\tilde{t}_{1}} m_{\tilde{t}_{2}}}$, so that in the light top squark region (small values of $m_{0}$ ) the scenario would fail to describe the correct value of the Higgs mass. The region that successfully accommodates the Higgs mass would require going to large values of $t_{\beta}$ and large values of the lightest top squark masses (say, $15 \lesssim m_{0} /|\mu| \lesssim 25$ ), in which case all sfermions would be out of reach of the experimental LHC searches, and only the gluino could be discovered in the near future. A benchmark case is provided in Table I, where we have chosen $t_{\beta}=10$ and $m_{0}=12 \mu$, with a heavy spectrum of supersymmetric scalars and a heavy Higgs sector and where, of course, only the gluino is detectable at the LHC. The last column contains the prediction for the light Higgs mass $m_{h}$, for which we have used FeynHiggs from Refs. [31-38], with an estimated theoretical error $\Delta m_{h} \lesssim$ $1 \mathrm{GeV}[39]$.

\section{B. Nonuniversal Higgs masses}

In the previous section we have seen that in the case of UHM the only way to solve the EoM and describe the correct Higgs mass is with a very heavy squark mass spectrum, which is out of reach of LHC searches. In this section we show that one way of avoiding this feature is by imposing different unified masses at the high unification

\footnotetext{
${ }^{3}$ The only constraint in the present scenario would be of course imposing the lightest neutralino to be the LSP, i.e., $m_{\tilde{t}_{1}}>m_{\tilde{\chi}_{1}^{0}}$.
}

scale $M$ for squarks and sleptons, and the Higgs sector. In particular, we introduce the boundary conditions at the scale $M$ given by

$$
\begin{aligned}
m_{Q}^{0} & =m_{U}^{0}=m_{D}^{0}=m_{L}^{0}=m_{E}^{0} \equiv m_{0}, \\
m_{H_{U}}^{0} & =m_{H_{D}}^{0} \equiv m_{H} .
\end{aligned}
$$

\section{Universal gaugino masses}

We first consider the case of universal gaugino masses at the scale $M$ given by the boundary conditions in Eq. (4.7). In this case, the free parameters are $\left(m_{0}, m_{H}, A_{t}^{0}, t_{\beta}\right)$. Motivated by the purpose of describing the Higgs mass with a light spectrum as much as possible, we consider the case of large $t_{\beta}$ and fix $t_{\beta}=10$, which is large enough to contribute efficiently to the tree-level calculation of the Higgs mass and small enough to consistently allow the neglect of the bottom Yukawa coupling, which we do in our analytical computation. The resulting three parameters $\left(m_{0}, m_{H}, A_{t}^{0}\right)$ can be confronted with the EoM (2.2).

TABLE I. Benchmark supersymmetric spectrum for the parameter values $t_{\beta}=10$ and $m_{0} \simeq 12 \mu$. All masses are in TeV, except for the SM Higgs $(h)$ mass which is in GeV. Generation indices run as $a=1,2,3, i=1,2 . S U(2)_{L}$ doublets are indicated by $\tilde{Q}_{L}$ and $\tilde{\ell}_{L}$ for squarks and sleptons, respectively.

\begin{tabular}{lccccccccccc}
\hline \hline Field & $\tilde{t}_{1}$ & $\tilde{t}_{2}$ & $\tilde{b}_{L}, \tilde{Q}_{L}^{i}$ & $\tilde{u}_{R}^{i}$ & $\tilde{d}_{R}^{a}$ & $\tilde{\ell}_{L}^{a}$ & $\tilde{e}_{R}^{a}$ & $H^{0, \pm}, A$ & $\tilde{g}$ & $h$ \\
\hline Mass $(\mathrm{TeV})$ & 7.7 & 11.1 & 13.5 & 13.4 & 13.3 & 13.4 & 13.3 & 13.4 & 2.2 & 124
\end{tabular}

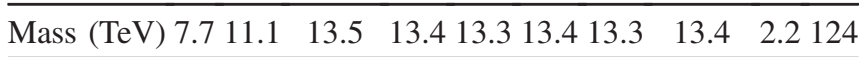



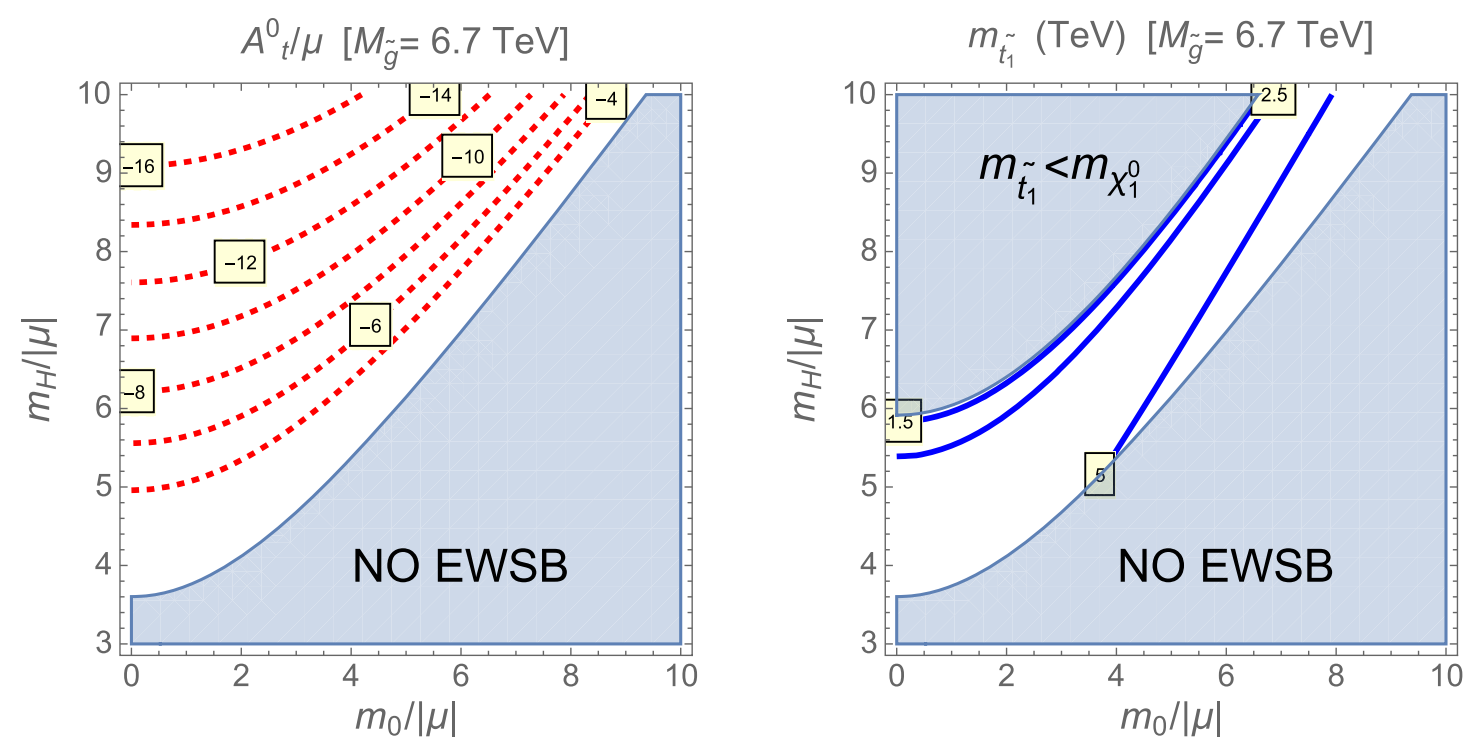

FIG. 5. Left: contour (dashed) lines of $A_{t}^{0} / \mu$ in the plane $\left(m_{0} /|\mu|, m_{H} /|\mu|\right)$ for $t_{\beta}=10$ satisfying the EoM (2.2) for universal gaugino masses. The shaded region is forbidden by the EWSB condition. Right: the same for the contour (solid) lines of the lightest top squark running mass $m_{\tilde{t}_{1}}$ in $\mathrm{TeV}$.

In the left panel of Fig. 5 we show the (dashed) contour lines of $A_{t}^{0} / \mu$ in the plane $\left(m_{0} /|\mu|, m_{H} /|\mu|\right)$, while the right panel shows the contour lines of $m_{\tilde{t}_{1}}$ in TeV. In both panels the lower shaded region is the forbidden region where there is no EWSB. In the right panel the upper shaded region is the forbidden region where $m_{\tilde{t}_{1}}<m_{\tilde{\chi}_{1}^{0}}$ and the lightest top squark would be the LSP. In the latter case we would need a lighter DM candidate, a condition that we do not explore in the present paper. As we can see in the right panel of Fig. 5, for small values of $m_{0}$ and values of $m_{H}$ near the upper shaded region the lightest top squark could be within reach of future LHC runs, and there are also relatively light states for the other squarks and sleptons. Moreover, in this region the top squark mixing parameter can be maximal, i.e., $\left|A_{t}-\mu / t_{\beta}\right| \simeq \sqrt{6} \sqrt{m_{\tilde{t}_{1}} m_{\tilde{t}_{2}}}$ and the Higgs mass can be easily accommodated by top squarks in the $\mathrm{TeV}$ region.

A benchmark model with maximal mixing is presented in Table II, where we give the tree level masses (in TeV) for the different scalars, and we have skipped the tiny splitting generated by the EWSB contribution. We can see that the lightest scalar is the third-generation right-handed slepton with a mass $\sim 1.2 \mathrm{TeV}$. The last column's prediction is

TABLE II. Benchmark supersymmetric spectrum for the parameter values $t_{\beta}=10, m_{0} \simeq 0$, and $m_{H} \simeq 6.3 \mu$. All masses are in $\mathrm{TeV}$, except for the SM Higgs mass $(h)$ which is in $\mathrm{GeV}$. Generation indices run as $a=1,2,3, i=1,2 . S U(2)_{L}$ doublets are indicated by $\tilde{Q}_{L}$ and $\tilde{\ell}_{L}$ for squarks and sleptons, respectively.

\begin{tabular}{lcccccccccccc}
\hline \hline Field & $\tilde{t}_{1}$ & $\tilde{t}_{2}$ & $\tilde{b}_{L}, \tilde{Q}_{L}^{i}$ & $\tilde{u}_{R}^{i}$ & $\tilde{d}_{R}^{a}$ & $\tilde{\ell}_{L}^{a}$ & $\tilde{e}_{R}^{a}$ & $H^{0, \pm}, A$ & $\tilde{g}$ & $h$ \\
\hline Mass (TeV) & 2.0 & 4.8 & 6.2 & 5.9 & 5.8 & 2.2 & 1.2 & 6.6 & 6.7 & 125 \\
\hline \hline
\end{tabular}

obtained from FeynHiggs in Refs. [31-38], with an estimated theoretical error $\Delta m_{h} \lesssim 2 \mathrm{GeV}$ [39].

\section{Nonuniversal gaugino masses}

Here we consider the case of nonuniversal gaugino masses at the scale $M$ corresponding to the boundary conditions in Eq. (4.9), still with free parameters given by $\left(m_{0}, m_{H}, A_{t}^{0}, t_{\beta}\right)$. As in the case of UHM, and mainly motivated by describing the correct value of the Higgs mass with a relatively light top squark spectrum, we fix $t_{\beta}=10$ so that the free parameters that we choose to satisfy the EoM (2.2) are $\left(m_{0}, m_{H}, A_{t}^{0}\right)$.

In the left panel of Fig. 6 we show the (dashed) contour lines of $A_{t}^{0} / \mu$ in the plane $\left(m_{0} /|\mu|, m_{H} /|\mu|\right)$, and in the right panel we show the contour lines of $m_{\tilde{t}_{1}}$ in TeV. In both panels the lower shaded region is the region where there is no EWSB. In the right panel the upper shaded region is the region where $m_{\tilde{t}_{1}}<m_{\tilde{\chi}_{1}^{0}}$ and such that the lightest top squark would be the LSP. For the smallest possible values of $m_{0}$ and $m_{H}$ consistent with EWSB, $m_{0} \simeq 1.9|\mu|$ and $m_{H} \simeq 1.5|\mu|$, the lightest top squark is as light as possible within the present model (lighter than in the case of universal gaugino masses), and the other squarks and heavy Higgses are also lighter than in the case of universal gaugino masses because of the smaller renormalization from the gluino mass and the smaller value of the common Higgs mass $m_{H}$, respectively, while sleptons are heavier because of the larger value of the common masses $m_{0}$. In this region the top squark mixing is near maximal, i.e., $\left|A_{t}-\mu / t_{\beta}\right| \simeq \sqrt{6} \sqrt{m_{\tilde{t}_{1}} m_{\tilde{t}_{2}}}$, and the Higgs mass can be easily accommodated. 

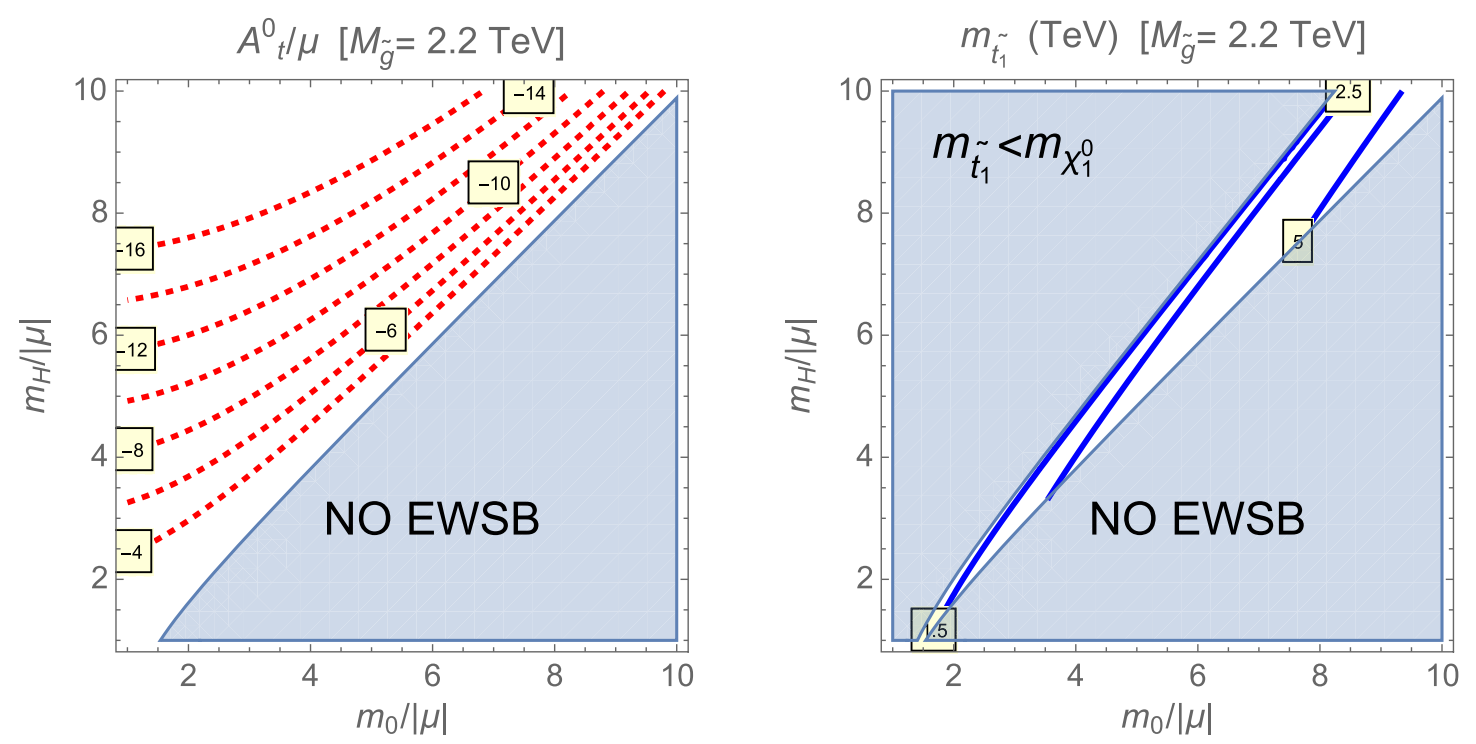

FIG. 6. Left: contour (dashed) lines of $A_{t}^{0} / \mu$ in the plane $\left(m_{0} /|\mu|, m_{H} /|\mu|\right)$ for $t_{\beta}=10$ satisfying the EoM (2.2) for nonuniversal gaugino masses. The shaded region is forbidden by the EWSB condition. Right: the same for the contour (solid) lines of the lightest top squark running mass $m_{\tilde{t}_{1}}$ in $\mathrm{TeV}$.

A benchmark model with maximal mixing is presented in Table III, where we give the tree level masses (in TeV) for the different scalars, and where (as done in Table II) we have skipped the tiny splitting generated by the EWSB contribution. We can see that the lightest scalar is the lightest top squark with a mass $\sim 1.6 \mathrm{TeV}$. The last column's prediction is obtained from the code FeynHiggs [31-38], with an estimated theoretical error $\Delta m_{h} \lesssim$ $2 \mathrm{GeV}$ [39].

\section{EXPERIMENTAL SIGNATURES}

In this section we comment on the different experimental signatures that the scenarios presented in this paper could have. The common feature and main motivation for this analysis has been a Higgsino doublet with a mass of $1.1 \mathrm{TeV}$ and with splitting among the lightest components of several GeV, as can be seen in Eq. (3.3). The production cross section of such a Higgsino is too low o be discovered at the LHC, but it can be produced at a $100 \mathrm{TeV}$ collider where the signal cross section is higher and it may be

TABLE III. Benchmark supersymmetric spectrum for the value of the gluino mass $M_{\tilde{g}}=2.2 \mathrm{TeV}$ and the parameter values $t_{\beta}=10, m_{0} \simeq 1.9 \mu$, and $m_{H} \simeq 1.5 \mu$. All masses are in $\mathrm{TeV}$, except for the SM Higgs mass $(h)$ which is in GeV. Generation indices run as $a=1,2,3, i=1,2 . S U(2)_{L}$ doublets are indicated by $\tilde{Q}_{L}$ and $\tilde{\ell}_{L}$ for squarks and sleptons, respectively.

\begin{tabular}{lccccccccccc}
\hline \hline Field & $\tilde{t}_{1}$ & $\tilde{t}_{2}$ & $\tilde{b}_{L}, \tilde{Q}_{L}^{i}$ & $\tilde{u}_{R}^{i}$ & $\tilde{d}_{R}^{a}$ & $\tilde{\ell}_{L}^{a}$ & $\tilde{e}_{R}^{a}$ & $H^{0, \pm}, A$ & $\tilde{g}$ & $h$ \\
\hline Mass $(\mathrm{TeV})$ & 1.57 & 3 & 3.5 & 2.9 & 2.8 & 3.1 & 2.4 & 2.8 & 2.2 & 124 \\
\hline \hline
\end{tabular}

possible to create sufficient amounts of highly boosted charginos and neutralinos for discovery [7,40,41].

A better option for discovering the Higgsino LSP is via dark matter direct-detection experiments [40,41]. The detection prospects strongly depend on the bino/wino admixture in the LSP, as that admixture controls the strength of the LSP-LSP-Higgs vertex that drives the spin-independent scattering rate off nuclei. ${ }^{4}$ The LSP for our benchmark points is around 99\% pure Higgsinoa result of the large wino/bino mass-so the spinindependent nuclear cross section for the benchmark range is of the order of a few $10^{-10} \mathrm{pb}$ [19]; thus, the whole range escapes the current limit from XENON-1T [26]. However, as shown in Ref. [19], an LSP Higgsino of this purity will be accessed in the next generation experiments, like XENON-nT or LZ [42].

Colored particles have higher cross sections and therefore can be easily produced at the LHC. In particular, the gluino has the largest cross sections, being a QCD octet. In scenarios of universal gaugino masses, they sit at a mass around or larger than $6.7 \mathrm{TeV}$, which is completely out of reach for the LHC. On the other hand, when one deviates from universal boundary conditions, the gluino mass can be almost a free parameter, and we have decided to put it at 2.2 TeV, which is the current LHC bound [43]. One feature of all spectra presented here is that there exists a heavy neutralino (mostly bino) $\chi_{3}^{0}$, whose mass is in between the gluino and Higgsino (LSP) masses; this fact will make the gluino decay, either directly to the LSP, or to $\chi_{3}^{0}$ which will

\footnotetext{
${ }^{4}$ The LSP Higgsino can also be detected via its spin-dependent scattering off nuclei, although the prospects there are not as good [19].
} 
subsequently decay to the LSP emitting a Higgs. This is not the usual assumption in experimental papers, where simplified models with the gluino decaying $100 \%$ to the LSP and jets are considered, and therefore all bounds should be reinterpreted for this particular case. Moreover, a characteristic signature of this kind of spectra would be a gluino, with different decay patterns, having Higgses in the cascade.

Top squarks are the second possibility for colored particles that could be discovered at the LHC. We have presented a typical benchmark spectrum with top squark masses of $\sim 1.6 \mathrm{TeV}$ in Table III. This "light" top squark evades the current bounds because the amount of missing energy is too small to trigger on the event [30]. In order to discover top squarks in the case where the splitting between the top squark and the LSP is around $500 \mathrm{GeV}$, we would need different techniques for this compressed situation, whereas in the benchmark shown in Table II the lightest top squark mass is $\sim 2 \mathrm{TeV}$, well above the current LHC bound [30]. This bound will be improved in the HL-LHC run, and hopefully it will reach a $2 \mathrm{TeV}$ top squark mass.

\section{CONCLUSION}

In this paper we focused on the appealing possibility that a nearly pure Higgsino with a mass $\sim 1.1 \mathrm{TeV}$ is the LSP, and therefore constitutes the DM of our Universe. We did so in the context of the MSSM, with supersymmetry breaking triggered by gravitational interactions at the (high) unification scale $M \simeq 2 \times 10^{16} \mathrm{GeV}$, in which case the $\mu$ parameter of the superpotential can be generated by Higgs interactions in the Kähler potential- the so-called GiudiceMasiero mechanism.

In particular, we considered two classes of models: i) models with universal Higgs masses, i.e., models where all scalar masses for sfermions and Higgses are equal at the unification scale, and ii) models with nonuniversal Higgs masses, i.e., models where the common mass in the Higgs sector is in general different from the common mass in the sfermion sector. In both classes of models we considered the cases of universal gaugino masses (i.e., all gaugino Majorana masses equal at the unification scale) and the cases where only the electroweakino masses are unified at the unification scale, while the gluino mass is put at its experimental lower bound.

In view of the strong experimental bounds on the mass of supersymmetric particles, we focused this work on the search for spectra that can be within reach of the future LHC runs or future high-energy colliders. Notice that, as the LSP is a Higgsino with a mass equal to $1.1 \mathrm{TeV}$, this means that all other supersymmetric particles are heavier and cannot be easily detected, in agreement with the recent experimental results on supersymmetric searches.

The conclusion for the neutralino/chargino sector is pretty general and model independent. The two lightest neutralinos and the lightest chargino are quasidegenerate at a mass $\sim 1.1 \mathrm{TeV}$, with a splitting of order a few $\mathrm{GeV}$. There is more freedom in the masses for the other electroweakinos but, using the bound from XENON1T as a guide, we have a heavy neutralino with a mass larger than $1.5 \mathrm{TeV}$ and the heaviest neutralino and chargino (almost degenerate) with a mass larger than $2.7 \mathrm{TeV}$.

For the sfermion sector, models with a universal Higgs mass have sfermions heavier than $\sim 10 \mathrm{TeV}$, depending on the gluino mass, and thus the only observable particle in this class of models can eventually be the gluino for models with nonuniversal gaugino masses. Models with a nonuniversal Higgs mass can have squarks in the (few) $\mathrm{TeV}$ range, depending on the gluino mass. For heavy gluinos the lightest sfermion is the right-handed stau. For light gluinos, the renormalization effects are milder, and there can exist $\mathrm{TeV}$ squarks in the third generation and sizable mixing among the third-generation squarks, such that the experimental value of the Higgs mass can be easily accomodated. In this case, the lightest sfermion is the lightest top squark.

In summary, the main purpose of this work was to determine spectra that could be within reach of future collider searches. In short, we found that-apart from the Higgsino which is the LSP - the lighter particles can be the gluino, the right-handed stau, and the lightest top squark. The prospects for discovering the Higgsino are at a future $100 \mathrm{TeV}$ collider and by direct search experiments at the XENON-nT or LZ experiments. The gluino can be discovered at the LHC if it is light enough. The fact that there is a neutralino (mostly bino) with a mass between the gluino and the LSP translates into additional decay channels for the gluino which should be incorporated into the codes used by the experimental programs. Finally, we have a model with the lightest top squark with a mass $\sim 1.3 \mathrm{TeV}$ which could be discovered in the HL-LHC run.

\section{ACKNOWLEDGMENTS}

The work of A. D. is partly supported by the National Science Foundation under Grant No. PHY-1820860. The work of M. Q. is partly supported by Spanish MINEICO under Grant No. FPA2017-88915-P, by the Catalan Government under Grant No. 2017SGR1069, and by Severo Ochoa Excellence Program of MINEICO under Grant No. SEV-2016-0588. 
[1] H. E. Haber and G. L. Kane, The search for supersymmetry: Probing physics beyond the Standard Model, Phys. Rep. 117, 75 (1985).

[2] S. P. Martin, A supersymmetry primer, Adv. Ser. Dir. High Energy Phys. 21, 1 (2010).

[3] P. A. Zyla et al. (Particle Data Group), Review of particle physics, Prog. Theor. Exp. Phys. 2020, 083 C01 (2020).

[4] G. Jungman, M. Kamionkowski, and K. Griest, Supersymmetric dark matter, Phys. Rep. 267, 195 (1996).

[5] N. Arkani-Hamed, A. Delgado, and G. F. Giudice, The welltempered neutralino, Nucl. Phys. B741, 108 (2006).

[6] H. Baer, V. Barger, and A. Mustafayev, Implications of a $125 \mathrm{GeV}$ Higgs scalar for LHC SUSY and neutralino dark matter searches, Phys. Rev. D 85, 075010 (2012).

[7] M. Low and L. T. Wang, Neutralino dark matter at $14 \mathrm{TeV}$ and 100 TeV, J. High Energy Phys. 08 (2014) 161.

[8] L. Roszkowski, E. M. Sessolo, and A. J. Williams, What next for the CMSSM and the NUHM: Improved prospects for superpartner and dark matter detection, J. High Energy Phys. 08 (2014) 067.

[9] L. Roszkowski, E. M. Sessolo, and A. J. Williams, Prospects for dark matter searches in the pMSSM, J. High Energy Phys. 02 (2015) 014.

[10] L. Roszkowski, E. M. Sessolo, and S. Trojanowski, WIMP dark matter candidates and searches-current status and future prospects, Rep. Prog. Phys. 81, 066201 (2018).

[11] C. Cheung, L. J. Hall, D. Pinner, and J. T. Ruderman, Prospects and blind spots for neutralino dark matter, J. High Energy Phys. 05 (2013) 100.

[12] S. P. Martin, Compressed supersymmetry and natural neutralino dark matter from top squark-mediated annihilation to top quarks, Phys. Rev. D 75, 115005 (2007).

[13] L. J. Hall, Y. Nomura, and S. Shirai, Spread supersymmetry with wino LSP: Gluino and dark matter signals, J. High Energy Phys. 01 (2013) 036.

[14] G. F. Giudice, T. Han, K. Wang, and L. T. Wang, Nearly degenerate gauginos and dark matter at the LHC, Phys. Rev. D 81, 115011 (2010).

[15] C. Boehm, P. S. B. Dev, A. Mazumdar, and E. Pukartas, Naturalness of light neutralino dark matter in pMSSM after LHC, XENON100 and Planck data, J. High Energy Phys. 06 (2013) 113.

[16] A. Fowlie, K. Kowalska, L. Roszkowski, E. M. Sessolo, and Y. L. S. Tsai, Dark matter and collider signatures of the MSSM, Phys. Rev. D 88, 055012 (2013).

[17] P. Huang, R. A. Roglans, D. D. Spiegel, Y. Sun, and C. E. M. Wagner, Constraints on supersymmetric dark matter for heavy scalar superpartners, Phys. Rev. D 95, 095021 (2017).

[18] M. Badziak, M. Olechowski, and P. Szczerbiak, Is welltempered neutralino in MSSM still alive after 2016 LUX results?, Phys. Lett. B 770, 226 (2017).

[19] K. Kowalska and E. M. Sessolo, The discreet charm of Higgsino dark matter-A pocket review, Adv. High Energy Phys. 2018, 1 (2018).

[20] G. Giudice and A. Masiero, A natural solution to the mu problem in supergravity theories, Phys. Lett. B 206, 480 (1988).

[21] A. Brignole, L. E. Ibanez, and C. Munoz, Soft supersymmetry breaking terms from supergravity and superstring models, Adv. Ser. Dir. High Energy Phys. 18, 125 (1998).
[22] K. Kowalska, L. Roszkowski, E. M. Sessolo, and S. Trojanowski, Low fine tuning in the MSSM with higgsino dark matter and unification constraints, J. High Energy Phys. 04 (2014) 166.

[23] D. Horton and G. G. Ross, Naturalness and focus points with non-universal gaugino masses, Nucl. Phys. B830, 221 (2010).

[24] A. Delgado, M. Quiros, and C. Wagner, General focus point in the MSSM, J. High Energy Phys. 04 (2014) 093.

[25] A. Delgado, M. Quiros, and C. Wagner, Focus point in the light stop scenario, Phys. Rev. D 90, 035011 (2014).

[26] E. Aprile et al. (XENON Collaboration), Dark Matter Search Results from a One Ton-Year Exposure of XENON1T, Phys. Rev. Lett. 121, 111302 (2018).

[27] S. AbdusSalam, B. Allanach, H. Dreiner, J. Ellis, U. Ellwanger, J. Gunion, S. Heinemeyer, M. Kraemer, M. Mangano, K. Olive, S. Rogerson, L. Roszkowski, M. Schlaffer, and G. Weiglein, Benchmark models, planes, lines and points for future SUSY searches at the LHC, Eur. Phys. J. C 71, 1835 (2011).

[28] A. Kaminska, G. G. Ross, and K. Schmidt-Hoberg, Nonuniversal gaugino masses and fine tuning implications for SUSY searches in the MSSM and the GNMSSM, J. High Energy Phys. 11 (2013) 209.

[29] P. Draper, G. Lee, and C. E. M. Wagner, Precise estimates of the Higgs mass in heavy supersymmetry, Phys. Rev. D 89, 055023 (2014).

[30] G. Aad et al. (ATLAS Collaboration), Search for a scalar partner of the top quark in the all-hadronic $t \bar{t}$ plus missing transverse momentum final state at $\sqrt{s}=$ $13 \mathrm{TeV}$ with the ATLAS detector, Eur. Phys. J. C 80, 737 (2020).

[31] S. Heinemeyer, W. Hollik, and G. Weiglein, FeynHiggs: A program for the calculation of the masses of the neutral $C P$ even Higgs bosons in the MSSM, Comput. Phys. Commun. 124, 76 (2000).

[32] S. Heinemeyer, W. Hollik, and G. Weiglein, The masses of the neutral $C P$-even Higgs bosons in the MSSM: Accurate analysis at the two loop level, Eur. Phys. J. C 9, 343 (1999).

[33] G. Degrassi, S. Heinemeyer, W. Hollik, P. Slavich, and G. Weiglein, Towards high precision predictions for the MSSM Higgs sector, Eur. Phys. J. C 28, 133 (2003).

[34] M. Frank, T. Hahn, S. Heinemeyer, W. Hollik, H. Rzehak, and G. Weiglein, The Higgs boson masses and mixings of the complex MSSM in the Feynman-diagrammatic approach, J. High Energy Phys. 02 (2007) 047.

[35] T. Hahn, S. Heinemeyer, W. Hollik, H. Rzehak, and G. Weiglein, High-Precision Predictions for the Light $C P$ Even Higgs Boson Mass of the Minimal Supersymmetric Standard Model, Phys. Rev. Lett. 112, 141801 (2014).

[36] H. Bahl and W. Hollik, Precise prediction for the light MSSM Higgs boson mass combining effective field theory and fixed-order calculations, Eur. Phys. J. C 76, 499 (2016).

[37] H. Bahl, S. Heinemeyer, W. Hollik, and G. Weiglein, Reconciling EFT and hybrid calculations of the light MSSM Higgs-boson mass, Eur. Phys. J. C 78, 57 (2018). 
[38] H. Bahl, T. Hahn, S. Heinemeyer, W. Hollik, S. Paßehr, H. Rzehak, and G. Weiglein, Precision calculations in the MSSM Higgs-boson sector with FeynHiggs 2.14, Comput. Phys. Commun. 249, 107099 (2020).

[39] H. Bahl, S. Heinemeyer, W. Hollik, and G. Weiglein, Theoretical uncertainties in the MSSM Higgs boson mass calculation, Eur. Phys. J. C 80, 497 (2020).

[40] J. Bramante, P. J. Fox, A. Martin, B. Ostdiek, T. Plehn, T. Schell, and M. Takeuchi, Relic neutralino surface at a 100 TeV collider, Phys. Rev. D 91, 054015 (2015).
[41] J. Bramante, N. Desai, P. Fox, A. Martin, B. Ostdiek, and T. Plehn, Towards the final word on neutralino dark matter, Phys. Rev. D 93, 063525 (2016).

[42] B. Mount et al., LUX-ZEPLIN (LZ) Technical Design Report, arXiv:1703.09144.

[43] G. Aad et al. (ATLAS Collaboration), Search for supersymmetry in final states with missing transverse momentum and multiple $b$-jets in proton-proton collisions at $\sqrt{s}=$ $13 \mathrm{TeV}$ with the ATLAS detector, Report No. ATLASCONF-2018-041. 